\title{
Signal Separation Based on Extended Least Squares
}

\author{
Liuyang Gao ${ }^{1,2}$, Song Chen ${ }^{1}$, Yinghua Tian ${ }^{1}$, Jiaying Yue ${ }^{1}$ \\ 1. National Digital Switching System Engineering and Technological R\&D Center(NDSC) \\ Zhengzhou, China \\ 2. Luoyang Electronic Equipment Testing Center(LEETC) \\ Jiyuan, China \\ gly03040104@163.com
}

\begin{abstract}
In this paper, a new signal separation method is proposed in this paper to solve the problem of poor separation effect of mixed signals in strong noise environment. Based on least squares (LS), the improved optimization model is extended by Kullback-Leibler dispersion to remove the random noise. Theoretical analysis and simulation experiments show that the algorithm proposed in this paper is superior to the existing algorithm in estimating the source signal, especially when the mixed signal is completely immersed in noise, the recovery effect of the source signal is more obvious than the existing algorithm.
\end{abstract}

Keywords- signal separation; least squares; Kullback-Leibler divergence.

\section{INTRODUCTION}

Separation of the source signals from the mixed signals is one of the research hotspots and emphases in signal processing [1]. In these problems, the source signals before mixing are unknown, while the mixing process is unknown. Signal separation extracts source signals from observed mixed signals and is widely used in digital communication [2], speech signal processing [3], medical diagnosis [4], image processing [5].

Nowadays, the methods of independent component analysis (ICA)[9], sparse component analysis (SCA) [6] and nonnegative matrix factorization (NMF) [7] are usually used for blind separation of mixed signals. The core idea of independent component analysis is to minimize the statistical relationship between each signal source [8]. FastICA algorithm is a typical ICA optimization algorithm. However, the ICA is not suitable for underdetermined systems and requires only one Gaussian signal in the source signal. Sparse component analysis can separate underdetermined systems, but the source signal is required to be sparse and not suitable for all mixed cases [9].

From a mathematical point of view, when the observed mixed signal matrix is positive, the NMF method makes it possible to solve the problem of signal separation. In fact, timedomain signals, such as speech signals, which may not necessarily satisfy the non-negative conditions, can be transformed to non-negative signals by signal transformation (such as FFT).

In 1999, D. Lee and H. Sueng proposed the NMF [10], which attracted great attention in the academic circles. NMF takes the advantages of simple calculation, fast factorization and obvious physical properties of the results, which has attracted great attention in academia. Various signal separation algorithms based on NMF have been studied and proposed [1113]. The basic NMF algorithm includes NMF algorithm based on Euclidean distance and NMF algorithm based on KullbackLeibler (KL).

NMF does not require statistical independence of the source, nor does it have non-Gaussian restrictions, and is also applicable to non-sparse cases. NMF is used for target signal separation, which can effectively separate several linear mixed signals, especially in the field of image signal separation [1417]. In the speech signal separation, the transformed non negative signals have also been applied to some extent.

In speech signal processing, the mixed speech signal will inevitably be affected by noise from all aspects, which makes it difficult to recognize the separated speech signal by least squares(LS) based NMF.

To solve the problem of poor separation effect of mixed signals in strong noise environment, a new LS based NMF algorithm is proposed by extending the KL divergence, which can effectively separate the source signals and interference noises, and its computational complexity is equivalent to that of the basic NMF algorithm without noise.

\section{LS BASED NMF}

The $n$ - dimension sources are mixed to produce the $m$ - dimension mixed observations. It may be assumed that the signal mixing mode is linear mixing. For convolutional mixing, it can be transformed into linear mixing by signal transformation. Suppose the unknown source signal matrix is $\mathbf{H} \in R^{n \times L}$; the observation data matrix is $\mathbf{V} \in R^{m \times L}$; the mixed matrix is $\mathbf{W} \in R^{m \times n}$. $L$ is the length of the signal data, $L$ ? $m, n$. The signal mixing model can be obtained as shown in Formula 1.

$$
\mathbf{V}=\mathbf{W H}
$$

The hybrid model is consistent with NMF: a nonnegative matrix is decomposed into the product of two nonnegative matrices, that is, for an arbitrary nonnegative matrix $\mathbf{V}$, the NMF algorithm can decompose it into the product of a nonnegative matrix $\mathbf{W}$ and a nonnegative matrix $\mathbf{H}$. Therefore, the extraction of the target signal from the mixed signal can be realized by NMF.

For the dimension of $\mathbf{W}, m \geq n$, and if $\|\mathbf{V}-\mathbf{W H}\|^{2}$ is the smallest, then the equation $\mathbf{V}=\mathbf{W H}$ has a unique solution, which is the least squares problem. The renewal formula of the optimal solution of $\mathbf{W}$ and $\mathbf{H}$ is: 


$$
\begin{aligned}
& \mathbf{W} \leftarrow \mathbf{V H}^{\dagger 2}=\mathbf{V} \mathbf{H}^{\mathrm{T}}\left(\mathbf{H} \mathbf{H}^{\mathrm{T}}\right)^{-1} \\
& \mathbf{H} \leftarrow \mathbf{W}^{\dagger 1} \mathbf{V}=\left(\mathbf{W}^{\mathrm{T}} \mathbf{W}\right)^{-1} \mathbf{W}^{\mathrm{T}} \mathbf{V}
\end{aligned}
$$

NMF algorithm based on the LS is directly applied to signal separation to obtain the LS-based NMF signal separation algorithm, abbreviated as LS-NMF.

\section{LS-NMF CONBINED WITH KULLBACK-LEIBLER DISPERSION}

When the mixed system is noisy, the signal mixing model under the noise condition is shown in the following expressions

$$
\mathbf{V}=\mathbf{W H}+\mathbf{N}
$$

Where $\mathbf{N}$ represents additive noise, $\mathbf{V}, \mathbf{N} \in R^{m \times L}, \mathbf{W} \in R^{m \times n}, \mathbf{H} \in R^{n \times L}$.

Correspondingly, for the noisy signal model $\mathbf{V}=\mathbf{W H}+\mathbf{N}$, the renewal formula of the optimal solution of $\mathbf{W}$ and $\mathbf{H}$ is:

$$
\begin{aligned}
& \mathbf{W} \leftarrow(\mathbf{V}-\mathbf{N}) \mathbf{H}^{\dagger 2}=(\mathbf{V}-\mathbf{N}) \mathbf{H}^{\mathrm{T}}\left(\mathbf{H H}^{\mathrm{T}}\right)^{-1} \\
& \mathbf{H} \leftarrow \mathbf{W}^{\dagger 1}(\mathbf{V}-\mathbf{N})=\left(\mathbf{W}^{\mathrm{T}} \mathbf{W}\right)^{-1} \mathbf{W}^{\mathrm{T}}(\mathbf{V}-\mathbf{N})
\end{aligned}
$$

Next we need to focus on solving the iteration of additive noise $\mathbf{N}$.

Correspondingly, the expanded KL divergence is:

$$
D=\sum_{i, j}\left((\mathbf{V})_{i j} \ln \frac{(\mathbf{V})_{i j}}{(\mathbf{W H})_{i j}+(\mathbf{N})_{i j}}-(\mathbf{V})_{i j}+(\mathbf{W H})_{i j}+(\mathbf{N})_{i j}\right)
$$

Under the condition of non-negative constraint, $(\mathbf{W})_{i j} \geq 0,(\mathbf{H})_{i j} \geq 0,(\mathbf{N})_{i j} \geq 0$.

For coefficient regularization, $\sum_{i}(\mathbf{W})_{i j}=1$. Therefore:

$$
\begin{aligned}
\sum_{i, j}\left(\sum_{r}(\mathbf{W})_{i r}(\mathbf{H})_{r j}+(\mathbf{N})_{i j}\right) & =\sum_{r, j}(\mathbf{H})_{r j} \sum_{i}(\mathbf{W})_{i r}+\sum_{i, j}(\mathbf{N})_{i j} \\
& =\sum_{r, j}(\mathbf{H})_{r j}+\sum_{i, j}(\mathbf{N})_{i j} \\
& =\sum_{i, j}(\mathbf{V})_{i j}
\end{aligned}
$$

So the optimization model can be described as:

$$
\begin{aligned}
{[\mathbf{W}, \mathbf{H}, \mathbf{N}]=} & \arg \min D(\mathbf{V} \| \mathbf{W H}+\mathbf{N}) \\
\text { s.t. } \quad & \sum_{i}(\mathbf{W})_{i j}=1, \\
& (\mathbf{W})_{i j} \geq 0, \\
& (\mathbf{H})_{i j} \geq 0, \\
& (\mathbf{N})_{i j} \geq 0, \\
& \sum_{r, j}(\mathbf{H})_{r j}+\sum_{i, j}(\mathbf{N})_{i j}=\sum_{i, j}(\mathbf{V})_{i j}
\end{aligned}
$$

The multiplication algorithm is used to iterate as follows:

$$
\begin{gathered}
(\mathbf{H})_{k j} \leftarrow(\mathbf{H})_{k j} \frac{\sum_{i}(\mathbf{W})_{i k}(\mathbf{V})_{i j} /\left((\mathbf{W H})_{i j}+(\mathbf{N})_{i j}\right)}{\sum_{i}(\mathbf{W})_{i k}} \\
(\mathbf{W})_{i k} \leftarrow(\mathbf{W})_{i k} \frac{\sum_{j}(\mathbf{H})_{k j}(\mathbf{V})_{i j} /\left((\mathbf{W H})_{i j}+(\mathbf{N})_{i j}\right)}{\sum_{j}(\mathbf{H})_{k j}} \\
(\mathbf{N})_{i j} \leftarrow(\mathbf{N})_{i j} \frac{(\mathbf{V})_{i j}}{(\mathbf{W H})_{i j}+(\mathbf{N})_{i j}}
\end{gathered}
$$

Considering the normalization of coefficients, the iterative process can be described as the following matrix form:

$$
\mathbf{N} \leftarrow \mathbf{N e}(\mathbf{V d}(\mathbf{W H}+\mathbf{N}))
$$

Where e is the Hadamard product of matrices, with $d$ representing the corresponding elements of matrix.

Combining 20, we can get the hybrid algorithm.

$$
\left\{\begin{array}{l}
\mathbf{W} \leftarrow(\mathbf{V}-\mathbf{N}) \mathbf{H}^{\mathrm{T}}\left(\mathbf{H} \mathbf{H}^{\mathrm{T}}\right)^{-1} \\
\mathbf{H} \leftarrow\left(\mathbf{W}^{\mathrm{T}} \mathbf{W}\right)^{-1} \mathbf{W}^{\mathrm{T}}(\mathbf{V}-\mathbf{N}) \\
\mathbf{N} \leftarrow \mathbf{N} \text { e }(\mathbf{V d}(\mathbf{W H}+\mathbf{N}))
\end{array}\right.
$$

\section{SIMULATION AND PERFORMANCE ANALYSIS}

\section{A. Experimental signal}

The experimental signals are taken from the TIMIT standard speech library. The four typical original signals is shown in Fig.1.
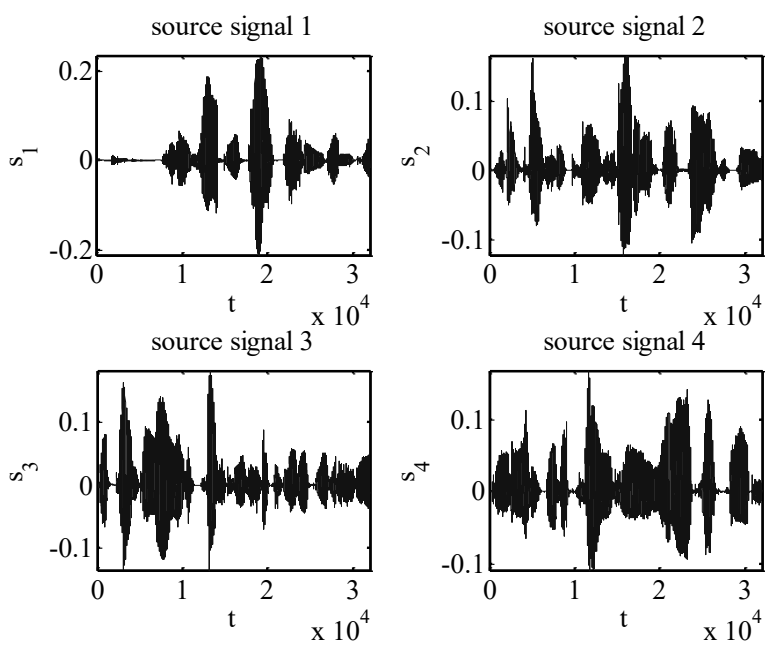

Fig.1 Original signals

A mixing matrix is randomly generated to mix the source signals. The mixed signals is shown in Fig.2. 

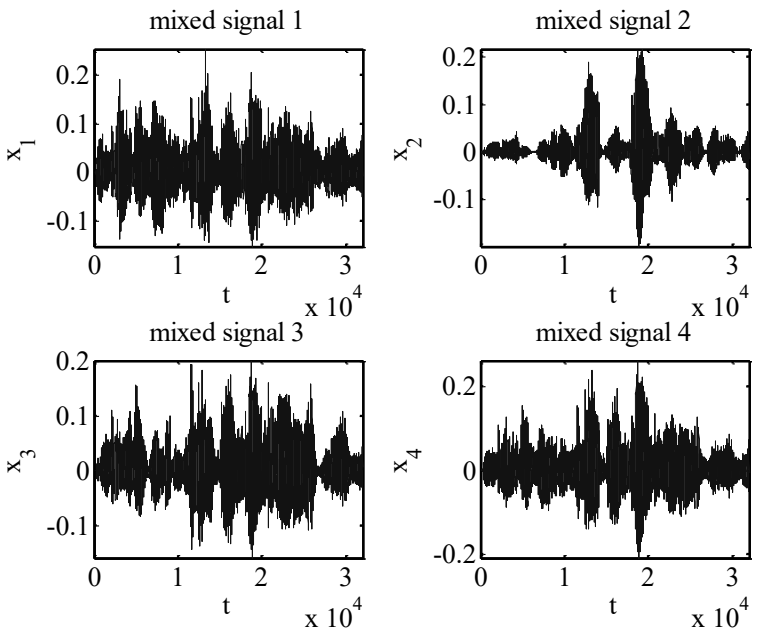

Fig.2 Mixed signals

Each mixed signal contains multiple signals, so multiple voices overlap and interfere with each other. In this case, the usual ICA or NMF algorithm can be used to effectively separate the signals. But in the noise environment, the separation effect will drop sharply. The mixed signal is shown in Fig. 5 when the signal-to-noise ratio is $0 \mathrm{~dB}$.
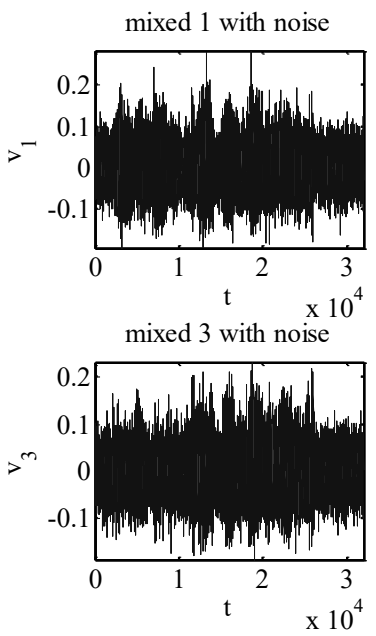
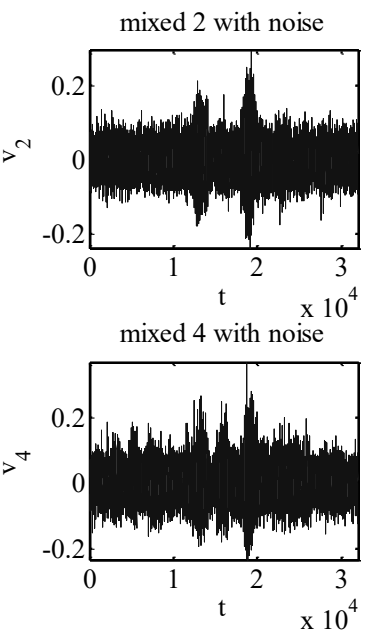

Fig.3 Mixed signal when the SNR is $0 \mathrm{~dB}$

Fig. 3 shows that the signal is almost completely annihilated when the SNR is $0 \mathrm{~dB}$, and the existence of speech can hardly be recognized. In this case, ICA or basic NMF method is used to separate the signal, but the separation effect is poor. The separation effect is directly shown by NMF method, as shown in Fig.4.
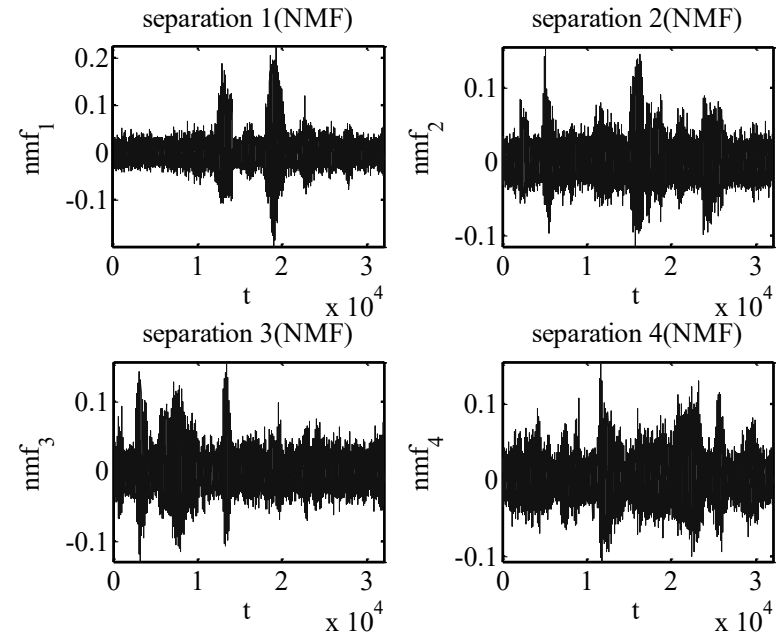

Fig.4 Separation by NMF method

Fig.4 shows that the signal can be separated to a certain extent by using NMF directly, but the separation is not thorough, and the influence of noise is very large, the separated speech is difficult to identify.

\section{B. Experimental results and performance analysis}

The unsupervised noise removal technique based on constrained NMF (UNMF) proposed in literature 18 is used to separate the source signal and identify the speech content. Noise can also be removed to a certain extent, but in the case of low signal-to-noise ratio, the effect of noise cancellation is limited, as shown in Fig.5.
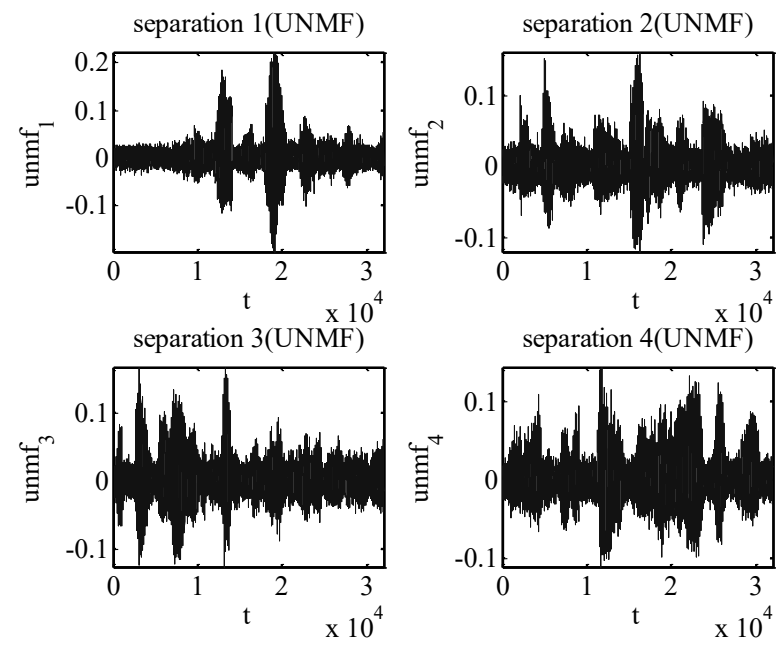

Fig. 5 Separation by UNMF method

The proposed extended LS algorithm can also eliminate noise more effectively, while the effect of signal separation is similar to that of the NNMF algorithm, as shown in Fig.6. 

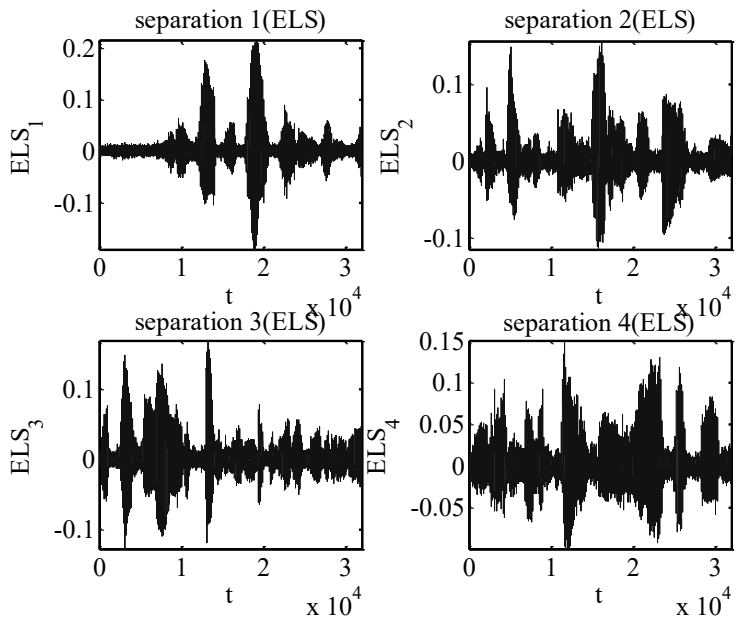

Fig.6 Separation by extended LS method

The experimental signals are taken from the TIMIT standard speech library. A mixing matrix is randomly generated to mix the source signals. Each mixed signal contains multiple signals, so multiple voices overlap and interfere with each other. In this case, the usual ICA or NMF algorithm can be used to effectively separate the signals. But in the noise environment, the separation effect will drop sharply.

Especially when the signal is almost completely annihilated when the SNR is $0 \mathrm{~dB}$, the existence of speech can hardly be recognized. In this case, ICA or basic NMF method is used to separate the signal, but the separation effect is poor. The unsupervised noise removal technique based on constrained NMF (UNMF) proposed in literature 18 is used to separate the source signal and identify the speech content. Noise can also be removed to a certain extent, but in the case of low signal-tonoise ratio, the effect of noise cancellation is limited. Using the proposed extended LS algorithm, the effect of signal separation and noise cancellation is better than that of UNMF algorithm.

\section{CONCLUSIONS}

Separation of mixed signals from noisy environments without prior conditions is one of the difficulties in blind signal separation. Based on the existing NMF algorithms, we propose an extended LS method for the separation of noisy mixed signals, in which an improved optimization model with random noise is designed and the KL divergence form is extended. A new target signal and noise estimation algorithm is proposed to overcome the shortcomings of existing methods in noise estimation. Theoretical analysis and simulation results show that the proposed ELS algorithm outperforms the existing algorithms in estimating the source signal. Especially when the signal and noise energy are equivalent and the mixed signal is completely obliterated in the noise, the extended LS algorithm has more obvious advantages than the existing algorithms.

\section{ACKNOWLEDGMENT}

This work was supported by the National Natural Science Foundation of China under Grant No. 61602511 and No. 61401513

\section{REFERENCES}

[1] G. Naik, W. Wang, Blind Source Separation: Advances in Theory, Algorithms and Applications. John Wiley \&Sons, Singapore, 2014.

[2] A. Veen, Blind separation of BPSK sources with residual carriers, Signal Processing, 1999, 73(1-2):67-79.

[3] A. Bell, T. Sejnowski, An information-maximization approach to blind separation and blind deconvolution. Neural Computation, 1995 , 7(6):1129-1159.

[4] R. Vigário, V. Jousmáki, M. Hämäläinen, Independent Componen Analysis for Identification of Artifacts in Magnetoencephalographic Recordings. Neural Information Processing Systems, 1997, 10:229-235.

[5] L, Guo, M, Garland, The use of entropy minimization for the solution of blind source separation problems in image analysis. Pattern Recognition, 2006, 39(6):1066-1073

[6] P. Comon, Independent component analysis-a new concept, Signal Process. 1994, 36(3), 287-314 .

[7] P. Bofill, M. Zibulevsky, Underdetermined blind source separation using sparse representations, Signal Process. 2001, 81(11):2353-2362.

[8] D. Lee, H. Sueng, Algorithms for Non-negative Matrix Factorization, Advances in Neural Information Processing Systems. Proceedings of the 2000 Conference, 556-562

[9] Y. Li, S. Amari and A, Cichocki, Underdetermined blind source separation based on Sparse Representation, Signal Processing, 2006 54(2):423-437.

[10] D. Lee, H. Sueng, Learning the parts of objects with nonnegative matrix factorization. Nature.1999, 401(6755):788

[11] K. Kwon, J. Shin, N. Kim, NMF-Based Speech Enhancement Using Bases Update. IEEE Signal Processing Letters.2015, 22(4):450-454.

[12] M. Sun, Y. Li, J. Gemmeke, Speech Enhancement Under Low SNR Conditions Via Noise Estimation Using Sparse and Low-Rank NMF with Kullback-Leibler Divergence. IEEE/ACM Transactions on Audio Speech \& Language Processing.2015, 23(7):1233-1242.

[13] S. Wood, J, Rouat, S. Dupont, Blind Speech Separation and Enhancement With GCC-NMF. IEEE/ACM Transactions on Audio Speech \& Language Processing.2017, 25(4):745-755.

[14] Y. Qian, S. Jia, Hyperspectral Unmixing via L1/2 Sparsity-Constrained Nonnegative Matrix Factorization. IEEE Transactions on Geoscience \& Remote Sensing.2011, 49(11):4282-4297.

[15] N. Wang, B Du, L. Zhang. An Endmember Dissimilarity Constrained Non-Negative Matrix Factorization Method for Hyperspectral Unmixing, IEEE Journal of Selected Topics in Applied Earth Observations \& Remote Sensing.2013, 6(2):554-569

[16] X. Lu, H. Wu, Yuan Y. Double Constrained NMF for Hyperspectral Unmixing. IEEE Transactions on Geoscience \& Remote Sensing.2014, 52(5):2746-2758.

[17] R. Rajabi, H. Ghassemian, Spectral Unmixing of Hyperspectral Imagery Using Multilayer NMF. IEEE Geoscience \& Remote Sensing Letters. 2014, 12(1):38-42.

[18] A. Vaghmare, C. Rao, Unsupervised noise removal technique based on constrained NMF. IET Signal Processing. 2017, 11(7):788-795. 